\title{
AN OVERALL CHANNEL MODEL FOR THE MOBILE USER OBJECTIVE SYSTEM INCLUDING IONOSPHERIC SCINTLLATION AND TERRESTRIAL MULTIPATH
}

\author{
M.J. Keskinen \\ Code 6750 \\ Naval Research Laboratory \\ Washington, DC 20375 \\ and \\ Per Kullstam \\ Paircom, Inc. \\ Springfield, VA 22153
}

\begin{abstract}
Using a channel impulse response function

formalism, an overall channel model which combines ionospheric scintillation fading and terrestrial multipath effects has been developed for the Mobile User Objective System (MUOS). For ionospheric scintillation fading we construct a channel impulse response function using a mutual coherence function. For terrestrial multipath we use a channel impulse response function based on a tapped-delay line model. We solve the combined model numerically using the Naval Research Laboratory Impulse Response Function Simulator code (NCIRFS) for several modulation waveforms for both frequencyselective and frequency nonselective fading conditions. We compute the delay spread for a simulated ground terminal in an urban and maritime fading environment. For an urban or maritime scintillation environment we find that the delay spread is a multiplicative combination of both multipath and ionospheric scintillation fading and can extend over several modulation periods. We present results for several waveforms and for combinations of fading and multipath environments.
\end{abstract}

\section{INTRODUCTION}

The Mobile User Objective System (MUOS) will provide satellite communications for a wide range of operational environments and for a large class of user terminals. The typical MUOS operational environments can be classified as land, ocean, and aeronauticalmobile satellite communications channels. Each of these channels has propagation conditions ranging from benign to stressed. For example, in the equatorial regions within approximately fifteen degrees of the magnetic equator, UHF transmissions can suffer both amplitude and phase scintillation and associated fading depending on geomagnetic conditions. In addition, in terrestrial environments, e.g., urban, suburban, maritime, strong multipath, either direct or diffuse, can also lead to signal fading. In the past ionospheric scintillation fading and terrestrial multipath fading have been studied separately. A coupled quantitative model has not been developed to date. In this paper we develop a coupled model for the combined effects of ionospheric scintillation and terrestrial multipath. We then apply the model to a terrestrial multipath environment undergoing ionospheric scintillation. Several quantities, e.g., delay spread, under the combined effects of ionospheric scintillation and terrestrial multipath, are computed. 


\section{MODEL}

If the transmitted signal is a modulated waveform $m(t)$, then the signal after propagation through the channel is the convolution of the transmitted modulation and the channel impulse response function [1]:

$$
u(r, t)=\int_{-\infty}^{\infty} m(t-\tau) h(r, t, \tau) d \tau
$$

where $u(r, t)$ is the received signal and $h(r, t, \tau)$ is the channel impulse response function. From this equation it is clear that the received signal at time $t$ and delay $\tau$ corresponds to transmitted modulation at time $t-\tau$. For the combined scintillation and terrestrial multipath problem we can generalize Eq. (1) as follows:

$$
u_{1}(t)=\int m(t-\tau) h_{\text {iono }}(t, \tau) d \tau
$$

where $u_{1}(t)$ is the received signal at the base of the ionosphere and $h_{\text {iono }}(t, \tau)$ is the channel impulse response function of the ionosphere. For the received signal on the ground we have :

$$
u_{2}(t)=\int u_{1}\left(t-\tau^{\prime}\right) h_{t e r r}\left(t, \tau^{\prime}\right) d \tau^{\prime}
$$

where $u_{2}(t)$ is the received signal on the ground and $h_{\text {eerr }}(t, \tau)$ is the channel impulse function for terrestrial multipath. Inserting Eq. (2) into (3) we have:

$$
u_{2}(t)=\iint m\left(t-\tau-\tau^{\prime}\right) h_{e f f}\left(t, \tau, \tau^{\prime}\right) d \tau d \tau^{\prime}
$$

where $h_{\text {eff }}\left(t, \tau, \tau^{\prime}\right)=h_{\text {iono }}\left(t-\tau, \tau^{\prime}\right) h_{\text {terr }}\left(t, \tau^{\prime}\right)$. We assume both channel impulse response functions are independent random variables. A similar approach is also taken in terrestrial cellular communications channel modeling. For the ionospheric channel impulse response function in scintillation conditions we proceed as follows. The channel response function can be Fourier transformed to the frequency domain:

$$
\begin{aligned}
& h_{\text {iono }}(r, \tau, t)=\iint \exp \left[i\left(k r-\omega_{D} t\right)\right] \\
& x \hat{h}_{\text {iono }}\left(k, \tau, \omega_{D}\right) d k_{\perp} d \omega_{D}
\end{aligned}
$$

Because we assume a statistically stationary ionospheric channel we must have delta function correlation in angle, delay, and Doppler:

$$
\begin{aligned}
& <\hat{h}_{\text {iono }}\left(k_{\perp}, \tau, \omega_{D}\right) \hat{h}_{\text {iono }}^{*}\left(k_{\perp}, \tau^{\prime}, \omega_{D}{ }^{\prime}\right)>= \\
& \delta\left(k_{\perp}-k_{\perp}^{\prime}\right) \delta\left(\omega_{D}-\omega_{D}^{\prime}\right) \delta\left(\tau-\tau^{\prime}\right) \\
& S\left(k_{\perp}, \tau, \omega_{D}\right)
\end{aligned}
$$

where $\mathrm{S}$ is the Fourier transform of the mutual coherence function $\Gamma$ given by :

$$
\begin{aligned}
& S\left(k_{\perp}, \tau, \omega_{D}\right)=\int \Gamma(r, \omega, t) \exp [-i(k r \\
& \left.\left.-\omega t-\omega_{D} t\right)\right] d r d \omega d t
\end{aligned}
$$

The quantity $S\left(k_{1}, \tau, \omega_{D}\right)$ is basically the mean signal power arriving within a finite angle of arrival interval, delay interval, and Doppler interval. The mutual coherence function $\Gamma$ is defined as $\Gamma(r, \omega, t)=<U(0,0,0) U^{*}(r, \omega, t)>$ where $\mathrm{U}(r, \omega, t)$ is a solution of the parabolic wave equation for propagation through the ionosphere with embedded turbulence [2]-[4]. The mutual coherence function can be written:

$$
\begin{gathered}
\Gamma(x, y, \omega, t)=\frac{\exp \left[-\frac{1}{2}\left(\frac{\sigma_{v} \omega_{0}}{\omega_{0}}\right)^{2}\right] \exp \left[-\frac{t^{2}}{\tau_{0}^{2}}\right]}{\left[1+\frac{i \omega \Lambda_{x}}{\omega_{c o h}}\right]^{1 / 2}\left[1+\frac{i \omega \Lambda_{y}}{\omega_{c o h}}\right]^{1 / 2}} \\
x \exp \left[\frac{-\left(x / l_{x}\right)^{2}}{i \omega \Lambda_{y}} \operatorname{loxp}_{c o h}\left[\frac{-\left(y / l_{y}\right)^{2}}{1+\frac{i \omega \Lambda_{x}}{\omega_{c o h}}}\right]\right.
\end{gathered}
$$

where we have the following definitions: 


$$
\begin{gathered}
\sigma_{\vartheta}{ }^{2}=r_{e}{ }^{2} \lambda^{2} L_{0} L\left\langle\Delta n_{e}{ }^{2}\right\rangle \\
\Lambda_{x}=\left[\frac{2 l_{x}^{4}}{l_{x}^{4}+l_{y}^{4}}\right]^{1 / 2} \\
\Lambda_{y}=\left[\frac{2 l_{y}^{4}}{l_{x}^{4}+l_{y}^{4}}\right]^{1 / 2} \\
l_{y}=\left[\frac{2}{\ln \left(L_{0} / l_{i}\right)}\right]^{1 / 2} \frac{L_{0}}{\sigma_{\vartheta}} \frac{z_{1}+z_{r}}{z_{t}} \\
\left.\omega_{c o h}=\frac{2}{2 c \ln \left(L_{0} / l_{i} / l_{i}\right)}\right]^{1 / 2} \frac{L_{0}}{\sigma_{\vartheta}} \frac{z_{t}+z_{r}}{\sigma_{\vartheta}{ }^{2}} \frac{L_{t}}{z_{t} z_{r}} \\
\tau_{0}=\left[\frac{2}{\ln \left(L_{0} / l_{i}\right.}\right]^{1 / 2} \frac{T_{0}}{\sigma_{\vartheta}}
\end{gathered}
$$

In the above $\sigma_{\varphi}^{2}, 1,1, \tau_{0}$, and $\omega_{\text {toh }}$ are the variance of phase after traversing the ionosphere, the decorrelation lengths in the $x$ and $y$ directions perpendicular to the propagation direction, decorrelation time, and coherence bandwidth, respectively. In addition, $\mathrm{L}_{0}, \mathrm{l}_{\mathrm{l}}, \mathrm{L}, \lambda, \Delta \mathrm{n}_{\mathrm{e}}, \mathrm{z}_{\mathrm{h}}, \mathrm{z}_{\mathrm{r}}$, are the outer scale of the ionospheric turbulence, the inner scale of the ionospheric turbulence, the ionospheric thickness, the transmitter wavelength, the average electron density fluctuation in the ionospheric turbulence, the distance from the transmitter to the ionospheric layer, and the distance from the receiver to the ionospheric layer.
We then approximate the Fourier component of the ionospheric channel impulse response function as:

$$
\hat{h}_{\text {iono }}\left(k_{\perp}, \tau, \omega_{D}\right)=\xi\left(k_{\perp}, \omega_{D}\right)\left[\widetilde{S}\left(k_{\perp}, \omega_{D}\right]^{1 / 2}\right.
$$

where

$$
\widetilde{S}\left(k_{\perp}, \omega_{D}\right)=\int S\left(k_{\perp}, \tau, \omega_{D}\right) d \tau
$$

and $\xi$ is a random variable. This then gives an expression for the ionospheric scintillation channel impulse response function:

$$
\begin{aligned}
& h_{\text {iono }}(r, \tau, t)=\int_{-\infty}^{\infty} \exp \left[i\left(k r_{0}-\omega_{D} t\right)\right. \\
& \xi\left(k, \omega_{D}\right)\left[\widetilde{S}\left(k, \omega_{D}\right)\right]^{1 / 2} d k d \omega_{D}
\end{aligned}
$$

For the terrestrial multipath environment we have both the direct and diffuse components [5]. We consider here only the diffuse component. For the terrestrial multipath channel impulse response function we take a tapped delay line model [1],[6]-[10]:

$$
h_{t e r r}(r, \tau, t)=\sum_{n} c_{n}(t) e^{i \theta_{n}} \delta\left(\tau-\tau_{n}\right)
$$

where $c_{n}, \tau_{n}$, and $\theta_{n}$ are the amplitude, delay, and phase shift of the multipath in the tapped delay line model. The $\tau_{\mathrm{n}}$ are taken from UHF multipath data [7]-[10].

For the signal modulation we take a rootraised cosine model:

$$
\begin{aligned}
& m(t)=\frac{4 \alpha}{\pi \sqrt{T}}\left[1-\left(\frac{4 \alpha t}{T}\right)^{2}\right]^{-1}\left[\cos \left[(1+\alpha) \frac{\pi t}{T}\right)+\right. \\
& \left.(4 \alpha t / T)^{-1} \cdot \sin ((1-\alpha) \pi t / T)\right]
\end{aligned}
$$


with $\alpha=0.22$. Here $T$ is the modulation period and $t$ is the time.

\section{RESULTS}

We solve Eq.(4) numerically using the Naval Research Laboratory Channel Impulse Response Function Simulator (NCIRFS) code. For the ionospheric channel we take a range of decorrelation times $\tau_{0}=0.3-1 \mathrm{sec}$ and coherence bandwidths $\omega_{\text {con }} / 2 \pi=0.5-3 \mathrm{MHz}$ which are representative of the ionospheric channel under scintillation fading conditions at equatorial and low latitudes. For the terrestrial multipath channel we take six delays that are representative of UHF multipath, i.e., $\tau_{n}=4$, $4.5,5.5 .5,6.6 .5 \mu \mathrm{sec}$ in an urban environment [7]-[10]. A similar approach is taken for the maritime multipath. For the modulation period we take $\mathrm{T}=1 \mu \mathrm{sec}$. Fig. 1 gives a sample of the predicted matched filter output amplitude as a function of delay and time. The total time duration in Fig. 1 is 20 decorrelation times or $6.2 \mathrm{sec}$. As can be noted from the figure the time delay is confined to a few pulsewidths for this set of initial conditions. Similar results are found for the combined ionospheric scintillation and maritime environment. In general we find that that the delay spread in the combined ionospheric and terrestrial channel is a multiplicative combination of the ionospheric delay spread and terrestrial multipath delay spread. We have also considered several different modulation schemes and found similar results. A more extensive set of results will be reported in a future publication.

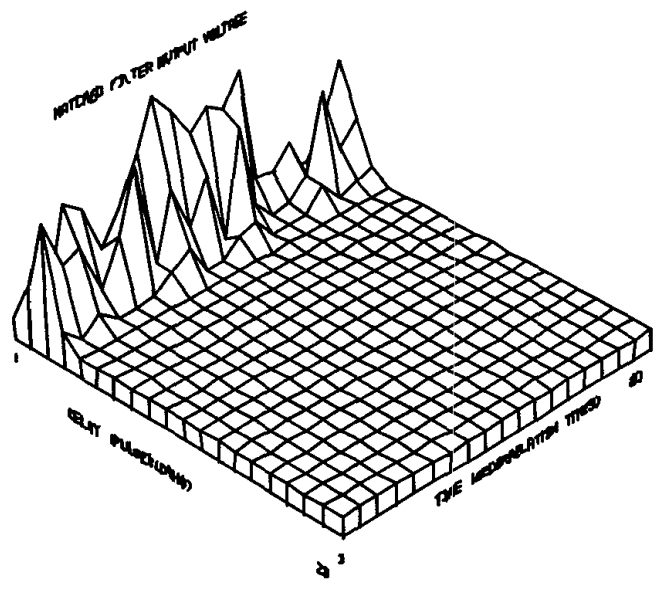

Fig. 1 Plot of matched filter amplitude vs. time and delay.

\section{SUMMARY}

We have developed a model for the combined effects of ionospheric scintillation fading and terrestrial multipath on an arbitrary waveform. The model is based on a channel impulse response formalism and can be initialized using a particular waveform modulation. We find that the delay spread for a combined ionospheric scintillation and terrestrial channel is a multiplicative combination of the delay spread due to the ionospheric channel and terrestrial channel. In the future we plan to study other channel parameter values, add other terrestrial channels, e.g, suburban, maritime, and consider other waveform modulations.

\section{ACKNOWLEDGMENTS}

We wish to acknowledgments useful discussions with CDR Jack Nicholson, Norm Baumgarten, Keith Kumm, Frank Tirpak, Bob Dresp, Fletcher Wicker, and Jim Loiselle. This work was supported by SPAWAR PMW146. 


\section{REFERENCES}

[1] J.G. Proakis, Digital Communications, Third Ed., New York, McGraw Hill, 1995.

[2] V.I. Tatarskii, The Effects of the Turbulent Atmosphere on Wave Propagation, National Technical Information Service, Springfield, VA 1971.

[3] A. Ishimaru, Wave Propagation and Scattering in Random Media, Academic, New York, 1978.

[4] K.C. Yeh and C.H. Liu, An investigation of temporal moments of stochastic waves, Radio Sci., 12, 671, 1977.

[5] S. Ohmori, H. Wakana, and S. Kawase, Mobile Satellite Communications, Artech, Boston, 1998.

[6] Y. Xie, and Y. Fang, A general statistical model for mobile satellite systems, IEEE

Trans. Veh Technol., 49, 744, 2000.

[7] E. Sousa, V. Jovanovic, and C. Daigneault, Delay spread measurements for the digital cellular channel in Toronto, IEEE Trans. Veh. Technol., 43, 837, 1994.

[8] T.S Rappaport, S.Y. Seidel, and R. Singh, $900 \mathrm{MHz}$ multipath propagation mesurements for U.S. digital cellular radiotelephones, IEEE Trans. Veh. Technol., 39, 132, 1990.

[9] G.L. Turin, F.D. Clapp, T.L. Johnston, S.B. Fine, and D. Lavry, A statistical model of urban multipath propagation, IEEE Trans. Veh. Technol., 21, 1, 1972.

[10] A. Zogg, Multipath delay spread in hilly region at $210 \mathrm{MHz}$, IEEE Trans. Veh.

Technol., 36, 184, 1987. 